Copyright by the AIP Publishing. Zwolak, M; Di Ventra, M, "DNA spintronics," Appl. Phys. Lett. 81, 925 (2002); http:// dx.doi.org/10.1063/1.1496504

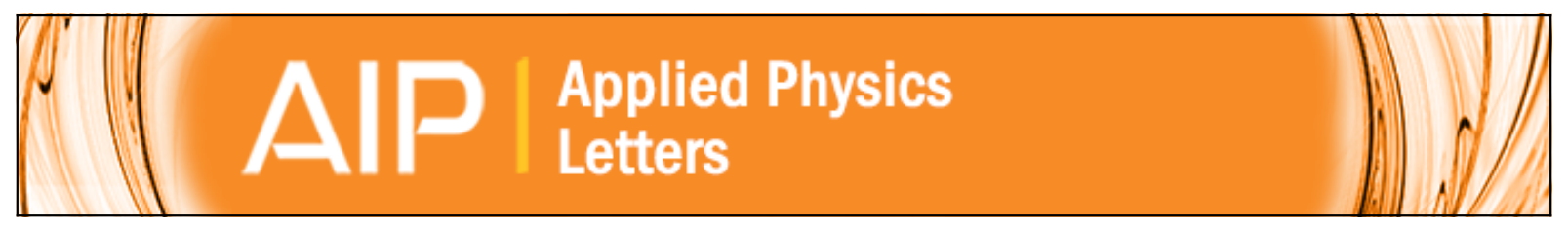

\title{
DNA spintronics
}

M. Zwolak and M. Di Ventra

Citation: Applied Physics Letters 81, 925 (2002); doi: 10.1063/1.1496504

View online: http://dx.doi.org/10.1063/1.1496504

View Table of Contents: http://scitation.aip.org/content/aip/journal/apl/81/5?ver=pdfcov

Published by the AIP Publishing

\section{Articles you may be interested in}

Double-stranded DNA field effect transistor and logical cells

J. Appl. Phys. 113, 074701 (2013); 10.1063/1.4792648

Detection of ferromagnetic particles using spin valve sensors

J. Appl. Phys. 100, 044909 (2006); 10.1063/1.2234537

Adsorption of DNA molecule and DNA Patterning on Si substrate

AIP Conf. Proc. 725, 3 (2004); 10.1063/1.1805372

DNA Nanotechnology

AIP Conf. Proc. 640, 63 (2002); 10.1063/1.1520078

Orthogonal pinning of two ferromagnetic layers in a synthetic spin valve

Appl. Phys. Lett. 80, 4576 (2002); 10.1063/1.1485106

\section{AlP}

\section{Create a profile.

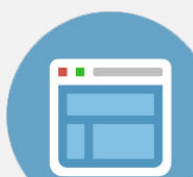 \\ Sign up today!}




\title{
DNA spintronics
}

\author{
M. Zwolak and M. Di Ventra ${ }^{a)}$ \\ Department of Physics, Virginia Polytechnic Institute and State University, Blacksburg, Virginia 24061-0435
}

(Received 30 April 2002; accepted for publication 3 June 2002)

We predict, using a tight-binding model, that spin-dependent transport can be observed in short DNA molecules sandwiched between ferromagnetic contacts. In particular, we show that a DNA spin valve can be realized with magnetoresistance values of as much as $26 \%$ for $\mathrm{Ni}$ and $16 \%$ for $\mathrm{Fe}$ contacts. Spin-dependent transport can broaden the possible applications of DNA as a component in molecular electronics and shed new light into the transport properties of this important biological molecule. (C) 2002 American Institute of Physics. [DOI: 10.1063/1.1496504]

Electronic transport in DNA has recently attracted considerable interest in view of its possible use in molecular electronics. ${ }^{1}$ Experiments indicate that DNA behaves as a metallic conductor, a semiconductor, or an insulator, according to different contacts, molecular lengths, and ambient surroundings. ${ }^{2}$ Direct measurements of electronic transport in short poly $(\mathrm{dG})$-poly $(\mathrm{dC})$ DNA molecules, in the $10 \mathrm{~nm}$ range, connected to metallic electrodes have revealed interesting nonlinear current-voltage $(I-V)$ characteristics. ${ }^{3}$ For voltages smaller than about $1 \mathrm{~V}$ an insulating gap is observed, while for larger voltages electric current can flow across the molecule. ${ }^{3}$ These results suggest that transport occurs when the electronic levels of the DNA molecule align with the quasi-Fermi levels of the electrodes. A theoretical account of such results has been recently provided by $\mathrm{Li}$ et al. by means of a homogeneous one-band tight-binding model for highest-occupied-molecular orbital-mediated charge transport. ${ }^{4}$ Quantitative agreement has been obtained by assuming partial electron dephasing on the guaninecytosine (GC) pairs. ${ }^{4}$

In this letter, we explore an extra degree of freedom in the transport properties of DNA, namely spin-dependent transport. In particular, we predict that spin-valve behavior could be observed in short DNA molecules of the type explored by Porath et al. ${ }^{3}$ when DNA is sandwiched between ferromagnetic contacts like $\mathrm{Ni}$ and $\mathrm{Fe}$. We show that magnetoresistance values of as much as $26 \%$ for $\mathrm{Ni}$ and $16 \%$ for $\mathrm{Fe}$ contacts can be observed. Apart from providing new insight into the fundamental mechanism of electronic transport in DNA, this study broadens the possible applications of DNA as a component in molecular electronics.

Following $\mathrm{Li}$ et al., ${ }^{4}$ we employ a one-band tightbinding model to simulate transport across a DNA molecule. The molecule is represented by a set of $N$ GC pairs. A schematic of the total system (DNA plus electrodes) investigated is shown in Fig. 1. The Hamiltonian is written as

$$
H=H_{M}+H_{L}+H_{R}+H_{\text {res }},
$$

where $H_{M}$ describes the DNA molecule, $H_{L}$ and $H_{R}$ the left and right electrodes, respectively, and $H_{\text {res }}$ describes electronic dephasing reservoirs. The latter terms are added as a

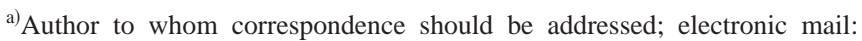
diventra@vt.edu
}

source of decoherence of the orbital component of the electronic wave function due to any type of inelastic scattering on each pair. Spin-flip effects are assumed to be negligible due to the very small spin-orbit coupling in DNA. This approximation seems to hold true for carbon nanotubes ${ }^{5}$ and phenyl molecules sandwiched between ferromagnetic contacts. $^{6}$

We treat the electrodes as semi-infinite one-dimensional tight-binding chains. The two spin configurations in the ferromagnetic contacts are represented by the different Fermi velocities for spin-up and spin-down populations, reflecting partially occupied $d$ bands for spin-down states and fully occupied $d$ bands for spin-up states. The Hamiltonian can be reduced to an effective Hamiltonian, $H_{\text {eff }}$, which is written as

$$
H_{\text {eff }}=H_{M}+\Sigma_{L}|1\rangle\left\langle 1\left|+\Sigma_{R}\right| N\right\rangle\left\langle N\left|+\sum_{j=1}^{N} \Sigma_{j}\right| j\right\rangle\langle j| .
$$

$\Sigma_{L(R)}$ and $\Sigma_{j}$ represent the interaction of the electrodes and reservoirs with the GC pair they interact with. These selfenergy corrections are written as

$$
\Sigma_{\mu}=\frac{V_{\mu}^{2}}{E-E_{\mu}-\sigma_{\mu}},
$$

where $\sigma_{\mu}=\left(E-E_{\mu}\right) / 2-i\left[\gamma_{\mu}^{2}-\left(E-E_{\mu}\right)^{2} / 4\right]^{1 / 2}$ and $\mu=L$, $R$, or $j$. The $V_{\mu}$ are the coupling strengths of the molecule to the electrodes and reservoirs (see Fig. 1). $E_{\mu}$ and $\gamma_{\mu}$ are, respectively, the site energies and hopping strengths of the electrodes and reservoir chains. Following $\mathrm{Li}$ et al., we take $4 \gamma_{j}$ as the band width of the dephasing reservoirs, assumed to be $5 \mathrm{eV}^{4}$ For the electrodes, we take

$$
\gamma_{L(R)}=\frac{\hbar v_{f, L(R)}}{2 a_{L(R)}}
$$

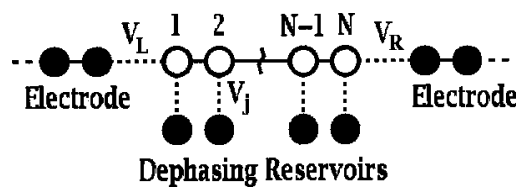

FIG. 1. Schematic of the system investigated. The DNA molecule is described by a one-dimensional tight-binding Hamiltonian. The interaction of the electrodes and dephasing reservoirs with the DNA molecule are described by the coupling parameters $V_{L(R)}$ and $V_{j}$, respectively. 
where $v_{f, L(R)}$ and $a_{L(R)}$ are the Fermi velocity and lattice parameter of the left (right) electrode. ${ }^{7}$

Assuming linear transport between any pair of electrodes/reservoirs, $\mu$ and $\nu(\mu, \nu=L, R$, and $j)$, the transmission coefficient is related to the Green's function ${ }^{8,9}$ by

$$
T_{\mu \nu}=4 \Delta_{\mu} \Delta_{\nu}\left|G_{\mu \nu}(E)\right|^{2}
$$

where $\Delta_{\mu(\nu)}=-\operatorname{Im} \Sigma_{\mu}$ and $G_{\mu \nu}(E)$ is the matrix element of the Green's function $G(E)$ between the molecular sites connected to the $\mu$ th and $\nu$ th electrodes/reservoirs. $G(E)$ is

$$
G(E)=\left(E I_{N}-H_{\text {eff }}\right)^{-1},
$$

where $I_{N}$ is the $N \times N$ identity matrix.

The total effective transmission coefficient, $T_{\text {eff }}(E)$, is constructed from the $T_{\mu \nu}:^{4}$

$$
T_{\mathrm{eff}}(E)=T_{L R}+\sum_{\mu, \nu=1}^{N} K_{\mu}^{(L)} W_{\mu \nu}^{-1} K_{\nu}^{(R)} .
$$

Here, $K_{\mu}^{(L)}=T_{L \mu}$ and $K_{\nu}^{(R)}=T_{\nu R}$. $W^{-1}$ is the inverse of $W$, with $\quad W_{\mu \nu}=\left(1-R_{\nu \nu}\right) \delta_{\mu \nu}-T_{\mu \nu}\left(1-\delta_{\mu \nu}\right), \quad R_{\nu \nu}=1$ $-\Sigma_{\mu \neq \nu} T_{\mu \nu}$.

The current can thus be written as

$$
I=\frac{2 e}{\hbar} \int_{-\infty}^{\infty} d E T_{\mathrm{eff}}(E)\left[f_{L}(E)-f_{R}(E)\right],
$$

where $f_{L(R)}=\left\{\exp \left[\left(E-\mu_{L(R)}\right) / k_{B} T\right]+1\right\}^{-1}$ is the Fermi function, and $\mu_{L(R)}$ the electrochemical potential of the left (right) electrodes.

We now consider transport between ferromagnetic contacts. Without spin scattering effects, we can treat transport of spin-up and spin-down electrons separately. We then add an additional index to the transmission coefficient, $T_{\text {eff }, i}(E)$, where $i$ represents an electronic state with spin up or down from the source that scatters into a spin-up or spin-down state in the drain.

For parallel alignment of the magnetization of the electrodes, spin-down electrons from the source are scattered only into spin-down states in the drain, likewise for spin-up electrons. The total transmission coefficient is thus

$$
T_{\text {eff }}(E)=T_{\text {eff, } \uparrow \uparrow}(E)+T_{\text {eff, } \downarrow \downarrow}(E) .
$$

For antiparallel alignment, spin-down electrons are scattered into spin-up states and the reverse for spin-up electrons. Since the two cases are symmetric in our model, the overall transmission coefficient can be written

$$
T_{\text {eff }}(E)=2 T_{\text {eff, } \uparrow \downarrow}(E) \text {. }
$$

Except for $\gamma_{L(R)}$, all parameters are taken to give a good fit to the experimental data of Porath et al. for a DNA molecule containing $30 \mathrm{GC}$ pairs making contact to nonmagnetic contacts. ${ }^{4}$ In particular, the coupling strength $V_{j}$ of the DNA molecule to the dephasing reservoirs is taken equal to 50 $\mathrm{meV} .{ }^{10}$ On the other hand, the hopping parameters $\gamma_{L(R)}$ differ for spin-down and spin-up electrons in the $\mathrm{Ni}$ and $\mathrm{Fe}$ contacts. We obtain their value from Eq. (4) using theoretical and experimental values for the Fermi velocities of Ni and $\mathrm{Fe}$, respectively. ${ }^{11,12}$ The hopping parameters for $\mathrm{Ni}$ are 0.7 and $0.2 \mathrm{eV}$ for spin-up and spin-down electrons, respectively. The corresponding values for $\mathrm{Fe}$ are $1.4 \mathrm{eV}$ for spin-up electrons and $0.6 \mathrm{eV}$ for spin-down electrons.
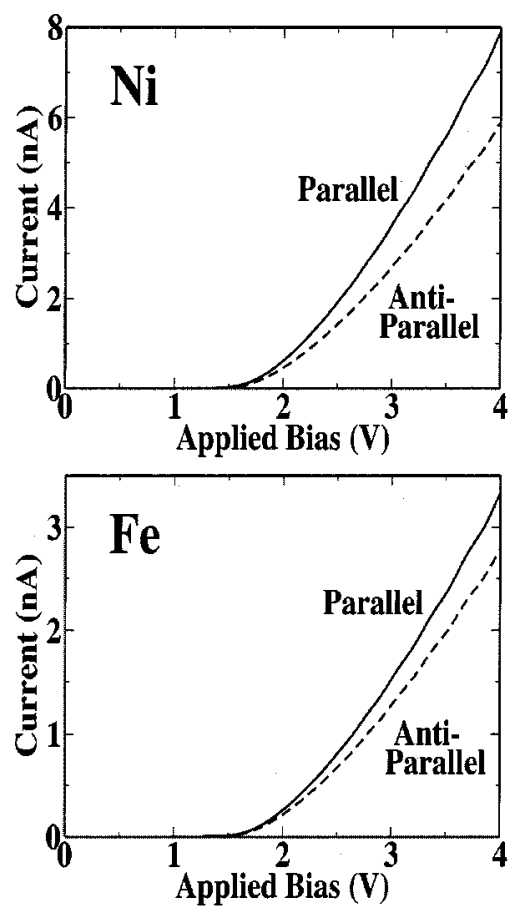

FIG. 2. $I-V$ curve at room temperature of a DNA molecule sandwiched between nickel (top panel) and iron (bottom panel) electrodes. Two cases are shown, one with parallel alignment of the magnetization of the two contacts, and the other with antiparallel alignment of the magnetization.

We assume that the coupling strengths at the contacts, $V_{L(R)}$, are the same for both spin-up and spin-down electrons. Electrons, for example, from the left electrode with spin-down magnetization, will have a larger transmission probability to scatter into the right-electrode states with the same magnetization than into states with opposite magnetization. This can be readily understood in terms of the different Fermi velocities of states with opposite magnetization. The velocity mismatch thus reduces the transmission probability. This mismatch is associated to the fact that, in the case of $\mathrm{Ni}$ and $\mathrm{Fe}$, the $d$ band is completely filled in the case of spin-up electrons. For the spin-down configuration, on the other hand, the $d$ band is only partially filled. ${ }^{5,6}$

The calculated $I-V$ curves at room temperature are shown in Fig. 2 for parallel and antiparallel alignment of the magnetization of $\mathrm{Ni}$ (top panel) and $\mathrm{Fe}$ (bottom panel) contacts. The figure shows that for both $\mathrm{Ni}$ and $\mathrm{Fe}$ the magnitude of the current decreases when scattering occurs from parallel to antiparallel contact configuration. The magnetoresistance, defined as $\left(R_{\text {anti }}-R_{\text {parallel }}\right) / R_{\text {anti }}$, is shown in Fig. 3, where $R_{\text {anti }}$ and $R_{\text {parallel }}$ are the resistances for the antiparallel and parallel spin configurations of the contacts, respectively. For $\mathrm{Ni}$, the magnitude of the magnetoresistance goes from a minimum of $12 \%$ for small biases to about $26 \%$ for biases larger than about $1.5 \mathrm{~V}$, which corresponds to the onset voltage for transport across the DNA molecule. For Fe, the magnetoresistance changes from about $11 \%$ to about $16 \%$. In both cases, however, the effect should be observable experimentally provided that spin scattering effects are small. The larger magnetoresistance for the $\mathrm{Ni}$ contacts can be understood in terms of the larger mismatch between spin-up and spin-down Fermi velocities (and, therefore, electrode hopping strengths) of $\mathrm{Ni}$ with respect to $\mathrm{Fe}$. It is also evident 


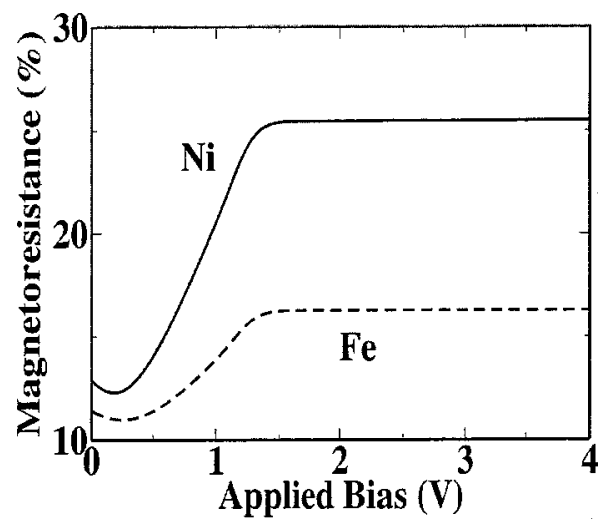

FIG. 3. Magnetoresistance as a function of bias for both $\mathrm{Ni}$ and Fe contacts at room temperature.

from Fig. 3 that the magnetoresistance is almost constant for applied voltages larger than $1.5 \mathrm{~V}$. This result can be rationalized by noting that for biases larger than $1.5 \mathrm{~V}$ the resistance for antiparallel magnetizations of the contacts increases faster than the corresponding resistance for parallel magnetizations (see Fig. 2).

In conclusion, we have shown that a DNA spin valve can be realized with magnetoresistance values of as much as $26 \%$ for $\mathrm{Ni}$ and $16 \%$ for $\mathrm{Fe}$ contacts. In both cases, spindependent transport should be experimentally observable. Spin-dependent transport can provide new insight into the fundamental mechanism of charge transfer in DNA, and broaden the possible applications of DNA as a component in molecular electronics.

We thank J. H. Schön for bringing to our attention the problem of spin-dependent transport in molecular structures. We also acknowledge support from the National Science Foundation Grant Nos. DMR-01-02277 and DMR-01-33075, and Carilion Biomedical Institute. Acknowledgment is also made to the Donors of The Petroleum Research Fund, ad- ministered by the American Chemical Society, for partial support of this research.

${ }^{1}$ See, e.g., C. Dekker and M. A. Ratner, Phys. World 14, 29 (2001).

${ }^{2}$ A. J. Storm, J. van Noort, S. de Vries, and C. Dekker, Appl. Phys. Lett. 79, 3881 (2001); H-W. Fink and C. Schönenberger, Nature (London) 398, 407 (1999); D. B. Hall, R. E. Holmlin, and J. K. Barton, ibid. 382, 731 (1996); E. Braun, Y. Eichen, U. Sivan, and G. Ben-Yoseph, ibid. 391, 775 (1999); A. P. Alivisatos, K. P. Johnson, T. E. Wilson, C. J. Loveth, M. P. Bruchex, and P. G. Schultz, ibid. 382, 609 (1996); B. Giese, J. Amaudrut, A. K. Kohler, M. Spormann, and S. Wessely, ibid. 412, 318 (2001); C. A. Mirkin, R. L. Letsinger, R. C. Mucic, and J. J. Storhoff, ibid. 382, 607 (1996); C. D. Mao, W. Q. Sun, Z. Y. Shen, and N. C. Seeman, ibid. 397, 144 (1999); E. Win-free, F. Liu, L. A. Wenzler, and N. C. Seeman, ibid. 394, 539 (1998); F. D. Lewis, T. F. Wu, Y. F. Zhang, R. L. Letsinger, S. R. Greenfield, and M. R. Wasielewski, Science 277, 673 (1997); A. Kasumov, M. Kociak, S. Gueron, B. Reulet, V. T. Volkov, D. V. Klinov, and H. Bouchiat, ibid. 291, 280 (2001); T. A. Taton, C. A. Mirkin, and R. L. Letsinger, ibid. 289, 1757 (2000); S. O. Kelley and J. K. Barton, ibid. 283, 375 (1999); F. D. Lewis, T. F. Wu, Y. F. Zhang, R. L. Letsinger, S. R. Greenfield, and M. R. Wasielewski, ibid. 277, 673 (1997); Y. Okahata, T. Kobayashi, K. Tanaka, and M. Shimomura, J. Am. Chem. Soc. 120, 6165 (1998); Y. A. Berlin, A. L. Burin, and M. A. Ratner, ibid. 123, 260 (2001); M. Bixon, B. Giese, S. Wessely, T. Langenbacher, M. E. Michel-Beyerle, and J. Jortner, Proc. Natl. Acad. Sci. U.S.A. 96, 11713 (1999); Y. A. Berlin, A. L. Burin, and M. A. Ratner, Chem. Phys. 275, 61 (2002).

${ }^{3}$ D. Porath, A. Bezryadin, S. de Vries, and C. Dekker, Nature (London) 403, 635 (2000).

${ }^{4}$ X. Li and Y. Yan, Appl. Phys. Lett. 79, 14 (2001).

${ }^{5}$ B. W. Alphenaar, K. Tsukagoshi, and M. Wagner, J. Appl. Phys. 89, 6863 (2001); B. Zhao, I. Monch, H. Vinzelberg, T. Muhl, and C. M. Schneider, Appl. Phys. Lett. 80, 3144 (2002).

${ }^{6}$ J. H. Schön, E. G. Emberly, and G. C. Kircznow, (unpublished).

${ }^{7}$ J. L. D'Amato and H. M. Pastawski, Phys. Rev. B 41, 11 (1990).

${ }^{8}$ V. Mujica, M. Kemp, and M. A. Ratner, J. Chem. Phys. 101, 6849 (1994).

${ }^{9}$ S. Datta, Electronic Transport in Mesoscopic Systems (Cambridge University Press, Cambridge, UK., 1995).

${ }^{10}$ Assuming that spin scattering processes are negligible, increased electronic dephasing changes the shape of the $I-V$ characteristics but affects only slightly the maximum value of the magnetoresistance.

${ }^{11}$ D. A. Papaconstantopoulos, Handbook of the Band Structure of Elemental Solids (Plenum, New York, 1986).

${ }^{12}$ Landolt-Börnstein, Numerical Data and Functional Relationships in Science and Technology, Vol. 19a, edited by K.-H. Hellwege and O. Madelung (Springer, Heidelberg, 1990). 